\title{
Cardiovascular magnetic resonance implementation in a newly established cardiomyopathies unit. Short-term results
}

\section{Utilidad de la cardiorresonancia magnética en una unidad de cardiopatías familiares de reciente implantación. Resultados a corto plazo}

\author{
Juan A. Requena-Ibáñez ${ }^{*}$, Jesús Piqueras-Flores ${ }^{1}$, Miguel Rienda-Moreno², Ramón Maseda-Uriza', \\ Pedro Pérez-Díaz ${ }^{1}$, and Raquel Frías-García ${ }^{1}$ \\ ${ }^{1}$ Department of Cardiology; ${ }^{2}$ Department of Radiology, Hospital General de Ciudad Real, Ciudad Real, Spain
}

Cardiac magnetic resonance (CMR) imaging has become an essential technique in the study of cardiomyopathies. It has multiple advantages that explain its growing role in clinical practice. It offers high spatial and temporal resolution images, and comprehensively assesses cardiac function and anatomy. It does not require the use of ionizing radiation. It is an imaging technique with multiplane capacity, free of geometric assumptions, and allows studying the heart and the great vessels without the limitations inherent to acoustic windows ${ }^{1}$. At present, it is the most accurate and reproducible technique for the study of global and regional biventricular systolic function².

In addition, it can characterize myocardial tissue for carrying out an etiological approach and assessing the presence of myocardial fibrosis. All of this confer this procedure an undoubted value in the diagnosis and prognostic grading of this type of patients.

Our objective is to assess the usefulness of CMR in a recently established heart disease family unit. From January 2017 to October 2018, 283 patients were evaluated. In the cases that had no contraindications, in those where the results of other diagnostic tests were discordant or tissue characterization or more accurate ventricular function study was necessary, a CRM was requested. Availability of the resonance equipment was also decisive in this center. Finally, the procedure was carried out in 83 patients.

The suspected diagnosis was hypertrophic cardiomyopathy (32 patients) followed by the left ventricular non-compaction (17) and dilated cardiomyopathy (14). Arrhythmogenic cardiomyopathy (6), Marfan syndrome and aortic involvement (5), cardiac amyloidosis (3), and restrictive cardiomyopathy (1) were less common.

To calculate the volumes, mass, ejection fraction, and wall thickness, balanced steady-state free precession (b-SSFP) sequences were used and therefore are the main source of anatomical and functional information. The features that make b-SSFP sequences ideal to study the heart are its high acquisition rapidness, high signalto-noise ratio, and high native contrast (no contrast media required) between blood, myocardium, and fat.

To study the phenomenon of delayed enhancement, extracellularly distributed gadolinium contrast medium is required, which increases CMR sensitivity to detect lesions and improves tissue characterization.

Gadolinium is a metal with a high paramagnetic capacity and maximum neutron attraction capacity, which turns it into a good contrast agent. There are different gadolinium chelates in the market, with slight pharmacological

\section{Correspondence:}

*Juan A. Requena-lbáñez

E-mail: juan.antonio.requena.ib@gmail.com
Available online: 04-12-2020
Date of reception: 26-02-2019 Date of acceptance: 29-03-2020 DOI: 10.24875/ACME.M20000140
Arch Cardiol Mex (Eng). 2020;90(3):334-335
www.archivoscardiologia.com

Arch Cardiol Mex (Eng). 2020;90(3):334-335
www.archivoscardiologia.com

is is an open access article under the CC BY-NC-ND 
differences between each other. At this center in particular, meglumine gadoterate was used at a dose of $3 \mathrm{~mL} / \mathrm{kg}$, which has a macrocyclic structure and ionic charge, with both these features improving the stability of the molecule.

Gadolinium has an extracellular distribution and spreads freely through the capillaries. It accumulates in the interstitial space of areas where there is damage to cell membrane integrity or increased extracellular space, either due to necrosis or inflammation or fibrosis. This way, delayed enhancement represents contrast abnormal accumulation in a tissue (enhancement) that persists for a long period of time (delayed), about 25 min after contrast bolus intravenous injection.

For this, T1-enhanced sequences deriving from the above-mentioned (b-SSFP) were used, to which an inversion pulse capable of canceling the signal of the healthy myocardium (hypointense) was added, thus exacerbating the contrast with areas of pathological enhancement (hyperintense).

Of the total number of requested cases, 60 corresponded to males, 57 to index cases, and 26 to family study cases. Initial suspicion was confirmed with CMR in 63. The most common were the following: 27 patients with hypertrophic cardiomyopathy, 11 with dilated cardiomyopathy, 10 with non-compaction cardiomyopathy, and 5 with arrhythmogenic cardiomyopathy. On the other hand, CRM was normal in 11 subjects, which enabled final discharge of 8 patients, mainly due to ventricular hypertrabeculation with no criteria consistent with the left ventricular non-compaction, suspected arrhythmogenic cardiomyopathy without evidence of structural or functional alterations, and increased myocardial thickness with no criteria consistent with ventricular hypertrophy.

In 25 of the studies, delayed gadolinium enhancement was detected, and in two of them, the CRM result was decisive for automated cardioverter-defibrillator (AICD) implantation. It was one case of dilated cardiomyopathy with the left ventricular non-compaction and severe ventricular dysfunction, and one case of familial hypertrophic cardiomyopathy. The presence of delayed enhancement represents poorer prognosis and higher risk in multiple heart diseases ${ }^{3,4}$. Although the decision to implant an AICD currently depends on LVEF value ${ }^{5}$, CMR helped to improve risk stratification in these patients and therefore to better rationalize its implantation.

In general, patients with delayed gadolinium enhancement show a significantly lower ejection fraction (47 vs. $55 \%, p=0.014$ ). For this, the t-test for independent samples was used.
Finally, genetic testing was requested in 63 patients. All of them had a CRM pathological result (in particular, hypertrophic degree increased wall thickness). Most often identified mutations affected sarcomeric genes, 11 MYBPC3 (Arg502Gln, Arg495Gln, and Glu542GIn) and 5 with TTN Cys3529Ser. As it usually occurs in genetic studies, non-clearly causal mutations were also recognized (two mutations of uncertain significance and three mutations on cosegregation study).

In conclusion, the experience in this center agrees with CMR current trend of consolidation as a complementary imaging technique to echocardiography in the study of cardiomyopathies. CMR allows, together with an adequate clinical approach, narrowing down differential diagnosis, improving risk stratification, and guiding clinical decisions ${ }^{6}$.

\section{Funding}

None.

\section{Conflicts of interest}

There are no conflicts of interest to declare.

\section{Ethical disclosures}

Protection of human and animal subjects. The authors declare that no experiments were performed on humans or animals for this investigation.

Confidentiality of data. The authors declare that no patient data appear in this article.

Right to privacy and informed consent. The authors declare that no patient data appear in this article.

\section{References}

1. Grothues F, Smith GC, Moon JC, Bellenger NG, Collins P. Comparison of interstudy reproducibility of cardiovascular magnetic resonance with two-dimensional echocardiography in normal subjects and in patients with heart failure or left ventricular hypertrophy. Am J Cardiol. 2002;90:29-34

2. San Román JA, Candell-Riera J, Arnold R. Análisis cuantitativo de la fun-ción ventricular izquierda comoherramienta para la investigación clínica. Fundamentos y metodología. Rev Esp Cardiol. 2009;62(5):535-51. Elliott P, Andersson B, Arbustini E. Classification of the cardiomyopathies: a position statement from the European Society Of Cardiology Working Group on Myocardial and Pericardial Diseases. Eur Heart J. 2008;29(2):270-276

3. Heidary S, Patel H, Chung J. Quantitative tissue characterization of infarct core and border zone in patients with ischemic cardiomyopathy by magnetic resonance is associated with future cardiovascular events. $J$ Am Coll Cardiol. 2010;55(24):2762-2768.

4. Green JJ, Berger JS, Kramer CM, Salerno M. Prognostic value of late gadolinium enhancement in clinical outcomes for hypertrophic cardiom-yopathy. JACC Cardiovasc Imaging. 2012;5(4):370-377.

5. Elliott P. Cardiomyopathy. Diagnosis and management of dilated car-diomyopathy. Heart. 2000;84(1):106-112.

6. De Smet K, Verdries D, Tanaka K. MRI in the assessment of non is-chemic myocardial diseases. European J Radiol. 2012;81(7): 1546-1548. 